\title{
Interval Type-2 Fuzzy Vibration Control of Multiple-degree-of-freedom Structure Using Shape-memory-material Absorber
}

\author{
Chih-Jer Lin, ${ }^{1 *}$ Chun-Ying Lee, ${ }^{2 \dagger}$ Chih-Keng Chen, ${ }^{3}$ and Yong-Sheng $\mathrm{Li}^{1}$ \\ ${ }^{1}$ Graduate Institute of Automation Technology, National Taipei University of Technology, \\ 1, Sec. 3, Zhong-xiao E. Rd. Taipei 10608, Taiwan \\ ${ }^{2}$ Department of Mechanical Engineering, National Taipei University of Technology, \\ 1, Sec. 3, Zhong-xiao E. Rd. Taipei 10608, Taiwan \\ ${ }^{3}$ Department of Vehicle Engineering, National Taipei University of Technology, \\ 1, Sec. 3, Zhong-xiao E. Rd. Taipei 10608, Taiwan
}

(Received March 28, 2018; accepted August 30, 2018)

Keywords: type-2 fuzzy controller, shape memory alloys, shape memory polymer, tunable vibration absorber

A tunable vibration absorber (TVA) is developed in this study. The TVA is composed of a vibrator mass and two helical springs. The helical spring is made of shape memory alloy (SMA) and shape memory polymer (SMP). When an external current source is applied to the SMA + SMP helical spring, the temperature of the shape memory materials (SMMs) increases and the TVA's dynamic characteristics can be tuned for control on demand. Therefore, we can use the tunable property of the TVA to absorb the vibration of other structures, because Young's modulus of the SMA + SMP helical spring is controllable. However, the relationship between the applied current and the TVA stiffness is nonlinear. Therefore, to understand the vibration characteristic of the TVA, the experiments are designed to establish an expert database of the interval type-2 fuzzy controller, which will be used to describe the nonlinear mapping between the input and the output. In the case studies, the TVA is applied to suppress the vibrations of a planar structure with multiple degrees of freedom. The resulting experiments validated the ability of the proposed method of vibration control.

\section{Introduction}

Smart materials have been successfully utilized to suppress vibration for decades owing to their various physical properties. For semi-active and active vibration control applications, smart materials including magneto-rheological fluid (MRF), electro-rheological fluid (ERF), piezo-electrical ceramic (PZT), and shape memory alloy (SMA) are used as the material of the tunable vibration absorber (TVA) to achieve vibration control using their controllable material properties. For example, MRF and ERF are functional fluids that can change their stiffness and damping coefficients with the adjustment of the magnetic or electric field. For PZT materials,

\footnotetext{
*Corresponding author: e-mail: cjlin@ntut.edu.tw

†Present affiliation: Graduate Institute of Manufacturing Technology

https://doi.org/10.18494/SAM.2018.2040
} 
an electric charge is generated as it is deformed; this is called the piezoelectricity effect. The opposite of this phenomenon also holds true. If an electric field is applied to a piezoelectric material, deformation occurs. Therefore, some researchers use PZT materials to produce force or displacement to actively suppress vibration. The physical properties of SMAs can be changed by changing their temperature. To enhance the performance of TVAs, semi-active and active designs were developed for various applications. ${ }^{(1-5)}$ Some TVAs are designed so that their resonant frequencies can be tuned by adjusting their stiffness and/or degree of damping. To adjust the stiffness, one of the alternatives is to change the geometry using active actuators $^{(6-8)}$ and the other is to change the material properties of the constituents. ${ }^{(9-11)}$ The above TVAs are also called actively tuned vibration absorbers (ATVAs).

Among all ATVA designs, smart materials are usually used as the controllable actuator for changing the vibration behavior of the main structure. ${ }^{(12-14)}$ Some ATVA designs incorporate the SMA structural member to achieve vibration control; however, the problem of transformation nonlinearity due to hysteresis is usually encountered. The exact cause is that the forward and backward martensitic transformations occur along different actuation routes for most SMAs. To deal with this problem, the design in which the SMA is replaced with shape memory polymer (SMP) was proposed in our previous study. ${ }^{(4)}$ Because SMPs are materials that undergo the glass transition and recover large strain upon heating and cooling, the material property change does not show hysteresis because of the glass transition. Therefore, the material consisting of SMA + SMP is easier to control than the SMA. In our previous study, a cantilevered beam of SMA + SMP was used for the TVA design. However, the cantilevered beam configuration only provides deformation flexibility in lateral directions. To increase the controllability of the TVA for vibration applications with multiple degrees of freedom, the beam design is changed into a helical spring. ${ }^{(5)}$ The helical TVA has shown its applicability as a structural member in three degrees of freedom (DOFs) vibration reduction, but the controller design was not investigated in the previous study.

To improve the performance of the proposed TVA, a fuzzy controller and an adaptive semiactive controller are studied with respect to the suppression of the vibration of a planar structure. Because the characteristics of the SMP and SMA are governed by temperature, the real-time fuzzy control is investigated to establish the vibration-temperature mapping through vibration experiments. For the smart structure to have the suitable stiffness and damping properties as the external excitation appears, many control strategies have been proposed. ${ }^{(15)}$ In most of the literature, the structure is usually approximated as a linear system by linearization; however, the estimated model introduces modeling uncertainty to the controller design. Therefore, the fuzzy set theory has been studied over the past decades to deal with model uncertainties.

For decision-making problems, the performance of the controller is affected by model uncertainties, which appear in a number of different forms. Model uncertainties can be represented in two distinctly different forms, parametric uncertainty and unstructured uncertainty. For robust control, solving this problem means to understand the influence of uncertainties and design a controller with robustness to reduce the effects of model uncertainties and reject the external disturbance. However, most uncertainties involved in any problem are a result of some information deficiency, that is, information may be incomplete, 
imprecise, fragmentary, not fully reliable, vague, contradictory, or deficient in some other ways. Therefore, Zedeh proposed the fuzzy set theory in 1965 to deal with this problem and introduced the concept of "linguistic variables", which in this article equates to a variable defined as a fuzzy set in 1973. ${ }^{(16,17)}$ Mamdani and Assilian successfully applied the "IF-THEN" rule to automatic control based on the fuzzy linguistic approach in 1974. ${ }^{(18,19)}$ Even though type-2 fuzzy sets were proposed in 1976, type-1 fuzzy sets have usually been applied over the past three decades more often than type- 2 fuzzy sets owing to the complexity of the latter. As the measurement has some uncertainties, it is difficult to determine its exact value; using type-1 fuzzy sets make more sense than using crisp sets for fuzzy reasoning. However, for type-1 fuzzy sets, using an accurate membership function is not reasonable for model uncertainties, so another type of fuzzy set is necessary to handle these uncertainties, called type-2 fuzzy sets. ${ }^{(20,21)}$ Over this decade, more and more researchers have begun studying type-2 fuzzy sets for various applications, because type- 2 fuzzy sets can handle the uncertainties that type-1 fuzzy sets cannot deal with.

\section{TVA Using SMA + SMP Helical Springs}

With the machine operated at different speeds for different manufacturing tasks, the frequency of vibration excitation caused by eccentricity can be time-varying. The original vibration absorber design proposed by Frahm is effective only for a specific narrow frequency span. ${ }^{(22)}$ Therefore, the original design is effective for a structure suffering vibration in a narrow frequency range. To deal with structures subjected to excitation in a wide frequency range, Lee et al. proposed a novel TVA consisting of a hybrid shape memory spring and an SMP sleeve. The hybrid shape memory wire used to fabricate the helical spring was composed of a superelastic (SE) Ni-Ti core within a SMP tube sleeve, as shown in Fig. 1. ${ }^{(4,5)}$

A photograph of the proposed twin helical spring TVA is shown in Fig. $1 .{ }^{(5)}$ At the bottom of the TVA is a mass block that consists of an acrylic rectangular prism and a vibrator mass, whose weight and size are $36 \mathrm{~g}$ and $30 \times 15 \times 10 \mathrm{~mm}^{3}$, respectively. At the top of the TVA is another insulating acrylic block that is employed as the fixture for mounting the springs to the main structure. The ends of the helical springs are fixed to the two blocks with plastic screws. To control the temperature of the $\mathrm{Ni}-\mathrm{Ti}$ core, current is applied to the lead wires on the top. To

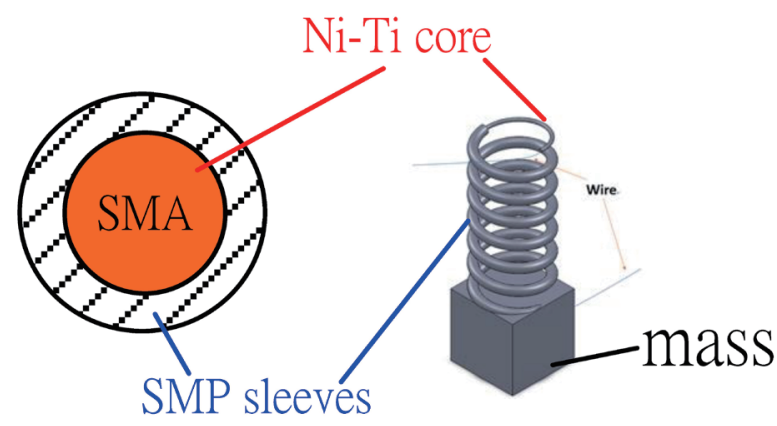

(a)

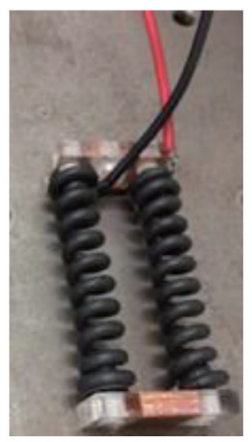

(b)

Fig. 1. (Color online) (a) Schematic diagram and cross section of the TVA. (b) Fabricated TVA. 
conduct the control current, the lead wires are connected to the spring ends at the mounting base (top) and the other ends of the springs are short-circuited with copper foil (bottom). The diameter of the Ni-Ti core is $0.45 \mathrm{~mm}$, and SMP has different outside diameters depending on the number of sleeve layers employed. The Ni-Ti wire is wound in a fixture with a helical groove and subsequently heat-treated at $450{ }^{\circ} \mathrm{C}$ for $2 \mathrm{~h}$ to form a helical spring. As a current is applied through the Ni-Ti core, the ohmic effect increases the temperature of the hybrid wire. However, the SE Ni-Ti wire is simply an electric conductor with no phase transformation, so only the SMP sleeves are significantly affected by the heating effect. Because different numbers of coils of the helical spring and different numbers of SMP sleeves wrapped around the Ni-Ti core cause the TVA stiffness to change in different ranges, in our previous work, we studied the mechanical properties of the hybrid shape memory wires for many case studies with different numbers of SMP sleeves and coils.

By changing the electric current through the SE core to change the temperature of the SMP sleeves, the natural frequency of the absorber can be controlled. In this study, the numbers of SMP sleeves and coils were determined from experimental results. According to the experimental results, the optimal design choice consists of three helical spring coils with four wrapped SMP sleeves. For different design parameters, the first six resonant frequencies of the TVA at 25 and $90{ }^{\circ} \mathrm{C}$ are listed in Table 1 . Among these designs with the different parameters, the third design can change the natural frequency with the widest range. Figure 2 shows that the first six resonant frequency ranges of the TVA are capable of changing the natural frequency

Table 1

Resonant frequencies.

\begin{tabular}{|c|c|c|c|c|c|c|}
\hline No. of coils & \multicolumn{2}{|c|}{5} & \multicolumn{2}{|c|}{3} & \multicolumn{2}{|c|}{3} \\
\hline No. of SMP sleeves & \multicolumn{2}{|c|}{3} & \multicolumn{2}{|c|}{3} & \multicolumn{2}{|c|}{4} \\
\hline Temperature $\left({ }^{\circ} \mathrm{C}\right)$ & 25 & 90 & 25 & 90 & 25 & 90 \\
\hline Mode 1 & 3.483 & 1.922 & 6.447 & 3.557 & 9.833 & 3.914 \\
\hline Mode 2 & 7.028 & 3.878 & 13.708 & 7.566 & 20.968 & 8.352 \\
\hline Mode 3 & 14.998 & 8.278 & 25.512 & 14.082 & 38.025 & 15.146 \\
\hline Mode 4 & 18.241 & 10.067 & 30.882 & 17.051 & 48.623 & 19.372 \\
\hline
\end{tabular}

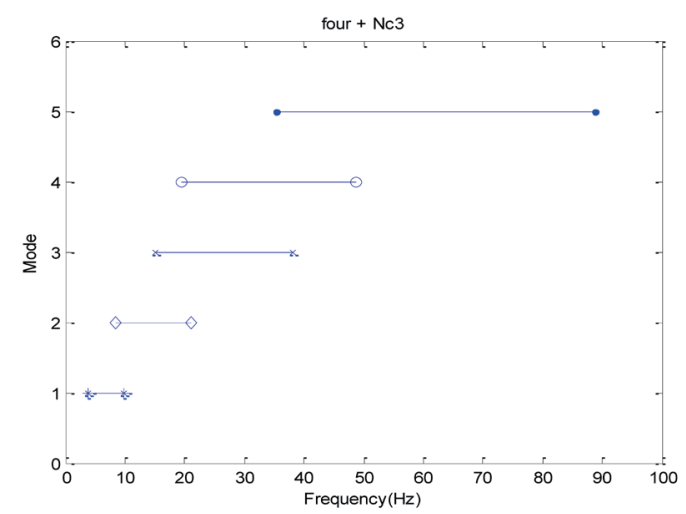

Fig. 2. (Color online) Frequency changes for the first six modes of the TVA with three helical spring coils and four wrapped SMP sleeves as the SE core temperature changes from 25 to $90{ }^{\circ} \mathrm{C}$. 
by more than $45 \%$ as the SE core temperature increases from 25 to $90{ }^{\circ} \mathrm{C}$. Therefore, the TVA with the third design will be used in the following experiments to change the structure's resonant frequency for suppressing vibration.

Theoretical formulation for absorber design is as follows. The proposed TVA is schematically shown in Fig. 3. The absorber is composed of two helical springs made of an SMA + SMP wire and an end mass block. The schematic diagram of the cross section of the SMA + SMP wire is shown in Fig. 1. The inner Ni-Ti core with the diameter $d_{A}$ was wrapped with the SMP sleeve on the outside to form the total outer diameter $d_{P}$. The simplified model for the design of this absorber was derived in our previous work. ${ }^{(5,6)}$ Therefore, the derivation process is not discussed in detail in this paper. Assume the mass block to be rigid and its center of gravity to be subjected to translations and rotations during vibration motion, such that the governing equation can be written as

$$
[M]\{\ddot{X}\}+[K]\{X\}=\{0\} .
$$

\section{Main Structure with Multidimensional Vibration Modes and Control Architecture}

To investigate vibration suppressing tasks of a planar structure using the TVA controlled by the proposed type-2 fuzzy controller, we adopt the self-designed experimental setup shown in Fig. 4 for control studies. The vibration of the main planar structure is produced by the two eccentric masses actuated by servomotors; the excitation vibration frequencies can be controlled by changing the rotation speed of the two motors. If the two motors are controlled at

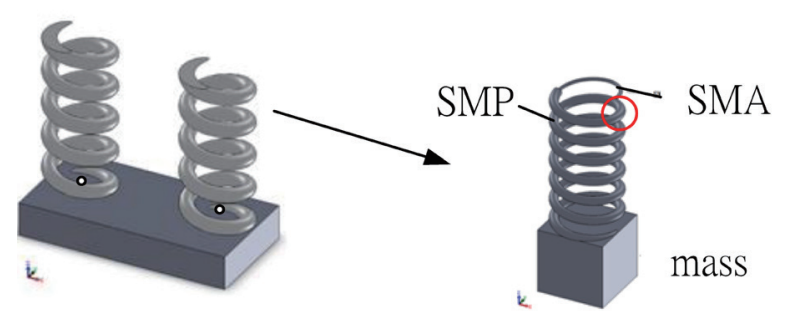

Fig. 3. (Color online) Schematic of the proposed tunable vibration absorber.

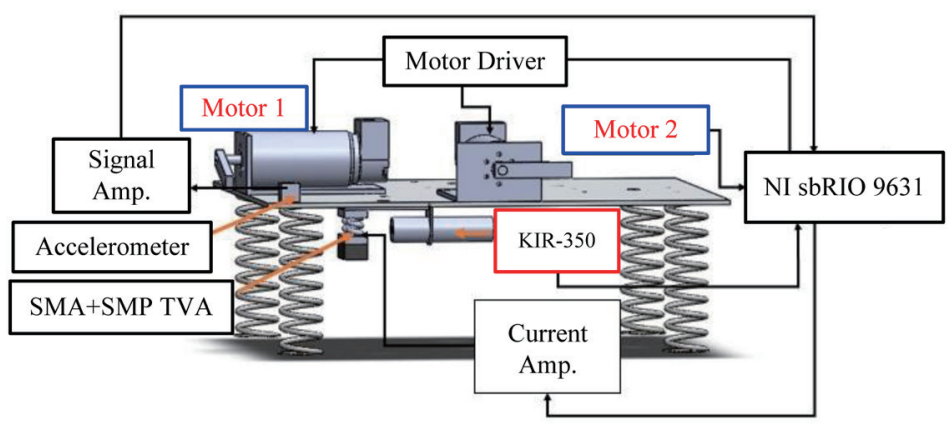

Fig. 4. (Color online) Schematic of the experimental setup. 
two different rotation speeds, the structure is affected by two exciting sources at two different frequencies. Therefore, this experimental system can be used to test the applicability of the proposed TVA for the structure with vibration modes in three dimensions. To perform vibration control using the proposed TVA, the measurements of the temperature of the absorber and the vibration of the main structure are very important for this system. To measure the temperature of the TVA for controller feedback, a noncontact infrared thermometer (KIR-350) is used in this system (as shown in Fig. 5). This sensor converts the infrared wavelength emitted from the target spot with respect to the specific temperature to a standard current signal output (4-20 $\mathrm{mA}$ ). It can measure -50 to a maximum of $350{ }^{\circ} \mathrm{C}$ with $\mathrm{RS}-485$ communication signal output. To measure from the vibration of the main structure, a 3 -axis accelerometer is used to detect the $Z$-axis acceleration of the main structure. To implement the proposed type- 2 fuzzy controller for suppressing the vibration in real time, a real-time embedded controller (NI sbRIO 9631) is used and the program of the controller is designed in Labview language. Then, the real-time application uses the field-programmable gate array (FPGA) reconfigurable embedded system (NI sbRIO 9631) with LabVIEW for real-time implementation.

\section{Experimental Results and Discussion}

The experimental setup for measuring and controlling the vibration absorber is presented in Fig. 4. The absorber was mounted perpendicular to the main structure. Therefore, the vibration modes associated with the lateral deformation of the helical springs could be more easily observed. During the vibration measurement, an excitation at a specified frequency was generated with the eccentric masses actuated by the servomotors. Then, the base excitation to the main structure was measured by the accelerometer (PCB 356A32). To understand the dynamic response of the main structure as an absorber at the specified temperature, a temperature controller is needed to hold the SMP's temperature at the desired value. In this research, a proportional-integral controller was studied and implemented using the sbRIO9631 embedded system. Figure 6 presents the block diagram of the whole control architecture for

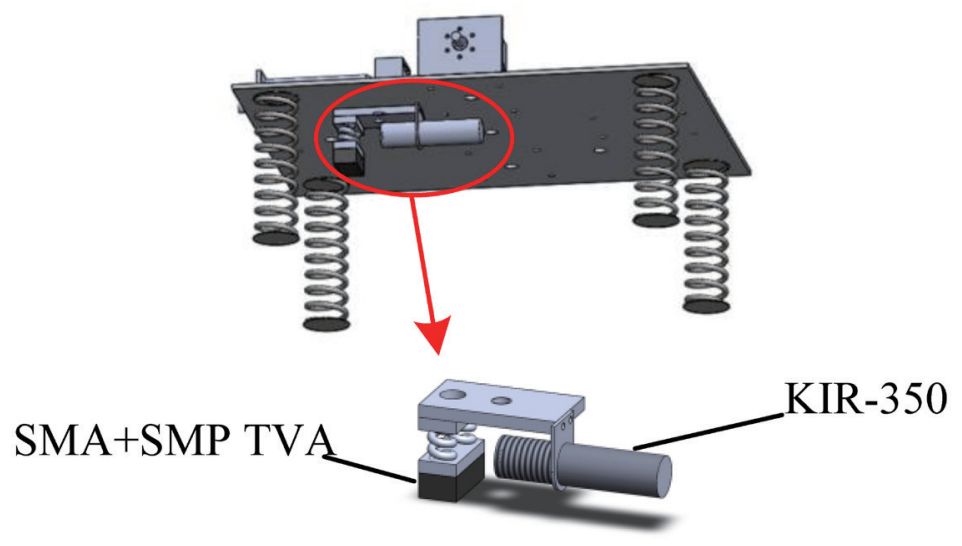

Fig. 5. (Color online) SMA + SMP TVA with the infrared thermometer (KIR-350) and its fixture. 
this TVA system. The acceleration of the main structure is transferred to root-mean-square (rms) and frequency values by the FFT method. These values are fed back to the fuzzy controller. After the fuzzy control produces the optimal temperature referring to the expert database, the desired temperature is fed back to the temperature controller.

For the input-output mapping relation of the fuzzy controller, the expert database can be obtained by investigating the relationship between the SMA temperature and the vibration response. To establish the fuzzy rule base for the controller, we designed experiments for the external vibrations at specified frequencies to obtain the optimal temperature of the TVA for vibrations at various frequencies. Table 2 shows the experimental results for the structure to which different currents were applied to produce the specified temperature on the SMA for the excitation at the frequencies of $10-20 \mathrm{~Hz}$. Then, a fuzzy controller is studied to establish mapping between the vibration situation and the temperature of the SMA. For the accelerometer of the $Z$-axis, the ratio of the output voltage to the acceleration is $100.5 \mathrm{mV} / \mathrm{g}$. Because the Vrms of the accelerometer's voltage for the $Z$-axis (Acceleration_z) is proportional to the vibration amplitude of the main structure, the controller should hold the TVA at the optimal temperature to reduce the vibration in accordance with the expert database in Table

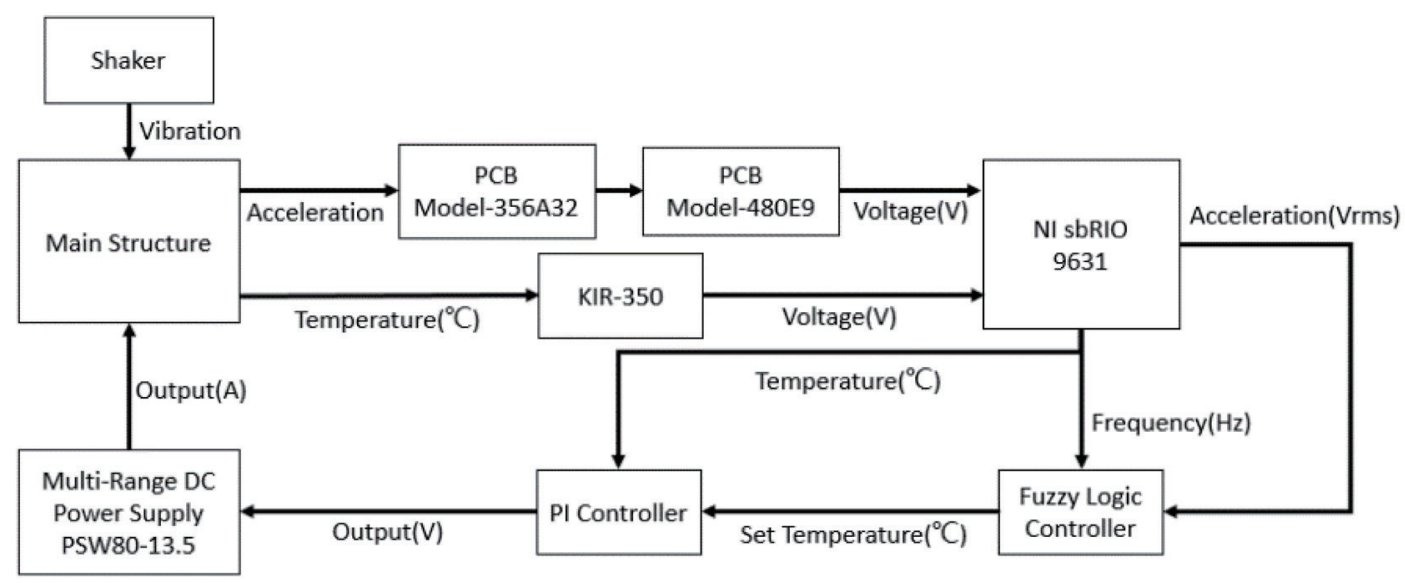

Fig. 6. Block diagram of the whole control architecture for our TVA system.

Table 2

(Color online) Experimental data for expert database of the fuzzy controller.

\begin{tabular}{lccccc}
\hline $\mathrm{mVrms}$ & $10 \mathrm{~Hz}$ & $17 \mathrm{~Hz}$ & $18 \mathrm{~Hz}$ & $19 \mathrm{~Hz}$ & $20 \mathrm{~Hz}$ \\
\hline $25^{\circ} \mathrm{C}$ & 31 & 45 & 96 & 314 & 120 \\
$30^{\circ} \mathrm{C}$ & 28 & 45 & 102 & 344 & 90 \\
$40^{\circ} \mathrm{C}$ & 32 & 51 & 114 & 51 & 65 \\
$50^{\circ} \mathrm{C}$ & 46 & 40 & 33 & 161 & 172 \\
$60^{\circ} \mathrm{C}$ & 62 & 29 & 50 & 202 & 299 \\
$70^{\circ} \mathrm{C}$ & 56 & 35 & 61 & 338 & 331 \\
$80^{\circ} \mathrm{C}$ & 64 & 36 & 63 & 230 & 395 \\
$90^{\circ} \mathrm{C}$ & 66 & 35 & 62 & 351 & 404 \\
\hline
\end{tabular}


2. For example, the SMA temperature should be maintained at $30^{\circ} \mathrm{C}$ to suppress the vibration efficiently as an excitation of $10 \mathrm{~Hz}$ is applied to the main structure. After recording the relationship between the temperature of the TVA and the acceleration of the main structure, the next step is to establish mapping between the optimal temperature and the vibration amplitude for the fuzzy controller on the basis of the experimental results.

\subsection{Type-2 fuzzy controller design for TVA}

An interval type-2 fuzzy logic system (FLS) is studied for vibration control in the presence of system modeling uncertainties. ${ }^{(10)}$ The type-2 fuzzy system has a structure similar to that of the type-1 fuzzy system, but the major difference is that the rule base has antecedents and consequents using type-2 fuzzy sets. A Gaussian function with a known standard deviation and a uniform weighting were used to represent a footprint of uncertainty, as shown in Figs. 7(a) and 7(b). Because of the use of such a uniform weighting, it is usually called an interval type-2 fuzzy set (IT2FS). For the IT2FS, a type reducer is needed to convert it to a type-1 fuzzy set before defuzzification is carried out. To obtain the output of the IT2FS, two fuzzy rules are needed, as shown in Tables 3 and 4. After obtaining the inference output, the type reducer and defuzzifier are used to obtain the input-output relationship. These two signals (Acceleration_z, $f$ ) are fed back to the proposed fuzzy controller. The optimal temperature reference is obtained from the input-output lookup relationship using the fuzzy controller. Then, the temperature controller induces the bipolar DC programmable current source (BP4610) to generate the current to actuate the TVA to suppress vibration. There are two excitations from the two motors, such that two peaks in the frequency response of the Acceleration_z can be obtained via the FFT, as shown in Fig. 8. Therefore, we proposed a switching control algorithm as follows:

$$
T_{o}=\frac{A_{1}}{A_{1}+A_{2}} \times T_{o 1}+\frac{A_{2}}{A_{1}+A_{2}} \times T_{o 2},
$$

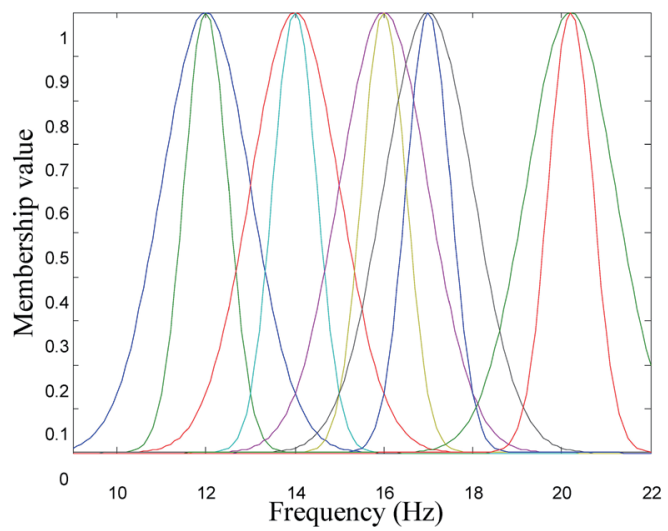

(a)

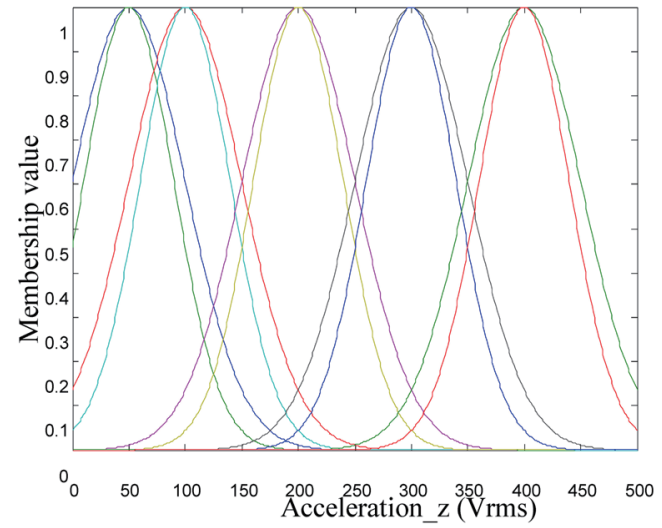

(b)

Fig. 7. (Color online) Type-2 fuzzy membership function for the input: (a) frequency and (b) rms values of the main structure's acceleration. 
Table 3

Right fuzzy rule base.

\begin{tabular}{lccccc}
\hline \multirow{2}{*}{ Frequency } & \multicolumn{5}{c}{ Acceleration_z } \\
\cline { 2 - 6 } & VS & S & M & B & VB \\
\hline VS & VS & VS & S & M & VS \\
S & S & S & S & M & S \\
M & M & S & M & B & B \\
B & S & VS & S & S & VB \\
VB & VS & VS & S & VS & VB \\
\hline
\end{tabular}

Table 4

Left fuzzy rule base.

\begin{tabular}{lccccc}
\hline \multirow{2}{*}{ Frequency } & \multicolumn{5}{c}{ Acceleration_z } \\
\cline { 2 - 6 } & VS & S & M & B & VB \\
\hline VS & VS & VS & S & M & VS \\
S & S & S & S & M & VS \\
M & M & S & M & B & B \\
B & S & S & S & S & VB \\
VB & S & VS & S & VS & VB \\
\hline
\end{tabular}

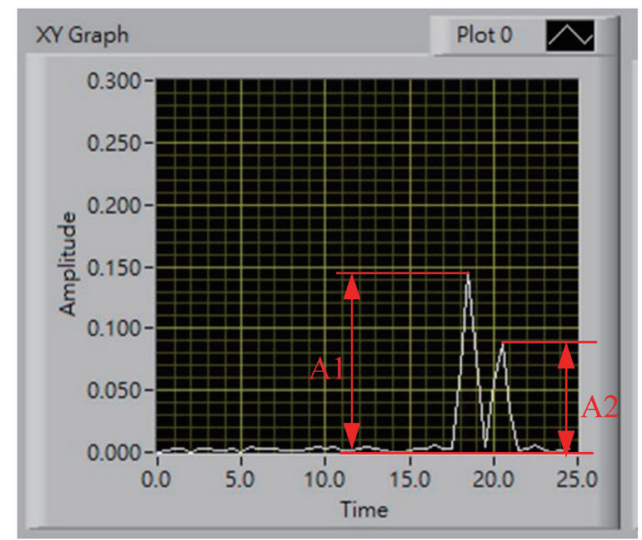

Fig. 8. (Color online) Vibration spectrum of the planar structure via FFT.

where $T_{o 1}$ is the output of fuzzy controller 1 for the input at the frequency of the first peak, $T_{o 2}$ is the output of fuzzy controller 2 for the input at the frequency of the second peak, $A_{1}$ is the amplitude of the first peak, and $A_{2}$ is the amplitude of the second peak. Figure 9 describes the proposed switching algorithm of the fuzzy controller.

\subsection{Experimental results and discussion}

To test the applicability of the proposed fuzzy controller, two cases are studied. The first case is to test the performance of the proposed controller in accordance with the signal external excitation produced by the first motor. The second case is to test the performance of the proposed controller in accordance with the dual external excitations produced by the two motors.

Case 1: First, considering the external excitation from Motor 1, we applied a vibration of 17 $\mathrm{Hz}$ to the main structure at $t=0 \mathrm{~s}$ and then changed the frequency of the excitation from 17 to $19 \mathrm{~Hz}$ at $t=105 \mathrm{~s}$. To compare the performance of the open-loop system with that of the closed-loop system, the proposed controller is turned on at $t=10 \mathrm{~s}$. Figure 10 shows the time response of the structure's acceleration for the real-time experiment; the result confirms that the proposed fuzzy controller can suppress vibration efficiently and rapidly. With the excitation of 


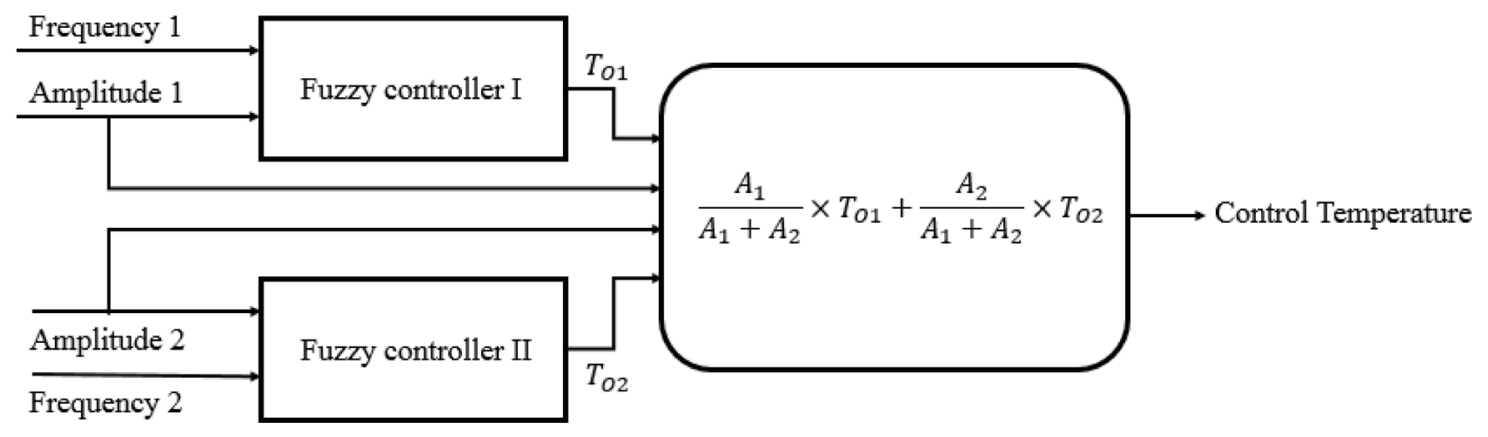

Fig. 9. Block diagram of the proposed control architecture for dual external excitations.
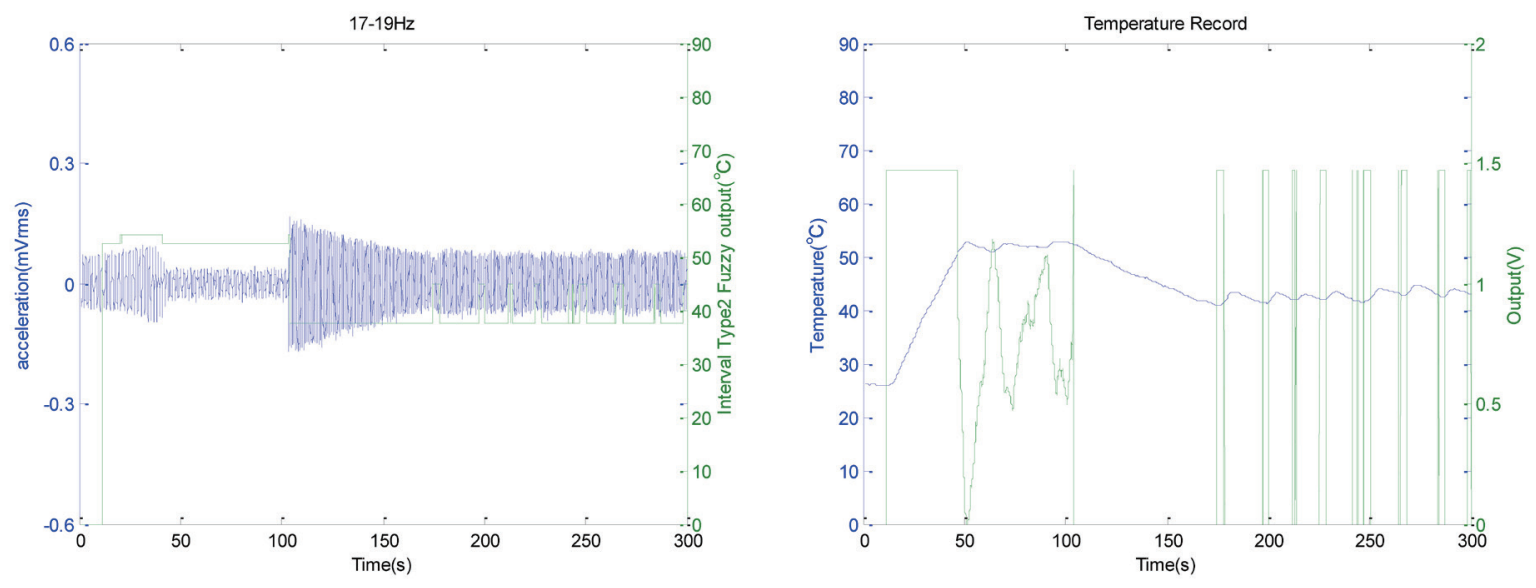

Fig. 10. (Color online) Experimental results obtained using the proposed controller in accordance with the external excitations of 17 and $19 \mathrm{~Hz}$.

$17 \mathrm{~Hz}$ applied to the planar structure, the output of the proposed fuzzy controller is about $52{ }^{\circ} \mathrm{C}$ and the vibration suppression rate is almost $52.2 \%$. With the excitation of $19 \mathrm{~Hz}$ applied to the planar structure, the output of the proposed fuzzy controller is about $36{ }^{\circ} \mathrm{C}$ and the vibration suppression rate is almost $58 \%$. These experimental results confirm that the proposed controller is effective for vibration reduction at frequencies of 17 and $19 \mathrm{~Hz}$.

Case 2: Consider the external excitations from Motors 1 and 2 simultaneously; the planar structure has the excitations of 17 and $19 \mathrm{~Hz}$, which are generated by Motors 1 and 2, respectively. To compare the performance of the open-loop system with that of the proposed controller, Fig. 11 shows the FFT spectra of the structure's accelerations for the proposed controller and open-loop system. These experimental results confirm that the proposed controller is effective for vibration reduction for multiple excitations at 17 and $19 \mathrm{~Hz}$. The vibration suppression rate in this case is almost $60.28 \%$. 


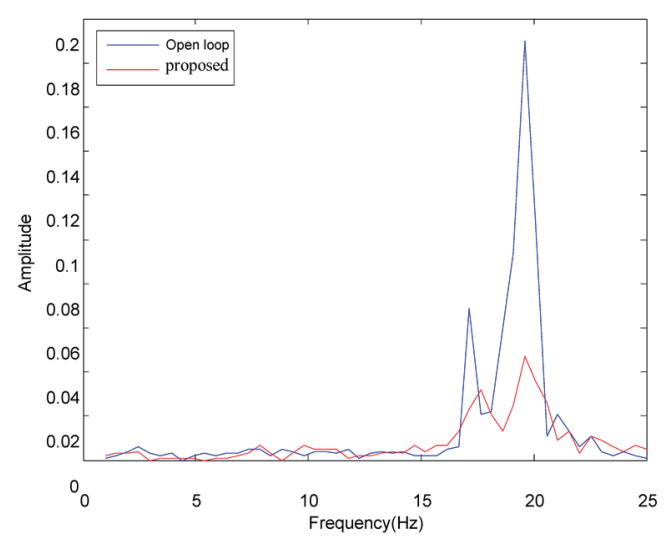

Fig. 11. (Color online) Vibration spectra of the proposed controller in accordance with the excitations of 17 and 19 $\mathrm{Hz}$.

\section{Conclusions}

An SMA + SMP TVA using a hybrid shape memory helical spring was presented to reduce the vibration of the main structure. The hybrid shape memory helical spring was made of two different shape memory materials (SMMs), namely, SMA and SMP. To determine the optimal temperature of the TVA for the excitation at a specified frequency, an expert database was first established in accordance with the relationship between the SMA temperature and the vibration response. Then, a type-2 fuzzy controller was designed to obtain the optimal temperature of the TVA for the main structure subjected to external excitation at specific frequencies. Fuzzy rules were determined from the RMS value of the acceleration for the main structure and its vibration frequency. To test the real-time implementation performance, the proposed fuzzy controller was implemented using the real-time embedded system (sbRIO 9631). Although there were model uncertainties of the TVA, the experimental results confirmed the applicability of the proposed controller for vibration reduction at multiple frequencies of 17 and $19 \mathrm{~Hz}$. With the excitation of $19 \mathrm{~Hz}$ applied to the main structure, the vibration suppression rate was almost $58 \%$. With multiple excitations of 17 and $19 \mathrm{~Hz}$ applied to the main structure, the vibration suppression rate was almost $60.28 \%$.

\section{Acknowledgments}

The authors would like to thank the National Science Council of the Republic of China, Taiwan, for financially supporting this research under Contract No. MOST 106-2221-E-027058.

\section{References}

1 M. J. Brennan: Shock Vib. 13 (2006) 531.

2 L. Kela and P. Vahaoja: Appl. Mech. Rev. 62 (2009) 060801-1. 
3 B. Tiseo, A. Concilio, S. Ameduri, and A. Gianvito: J. Theor. Appl. Mech. 48 (2010) 135.

4 C. Y. Lee, C. C. Chen, T. H. Yang, and C. J. Lin: J. Intell. Mater. Syst. Struct. 23 (2012) 1725.

5 C. Y. Lee and C. A. Pai: J. Intell. Mater. Syst. Struct. 27 (2016) 1047.

6 P. Bonello, M. J. Brennan, and S. J. Elliott: Smart Mater. Struct. 14 (2005) 1055.

7 B. Tiseo, A. Concilio, S Ameduri, and A. Gianvito: J. Theor. Appl. Mech. 48 (2010) 135.

8 A. K. Ghorbani-Tanha, M. Rahimian, and A. Noorzad: J. Eng. Mech. 137 (2011) 390.

9 C. J. Lin, C. Y. Lee, Y. Liu, and H. C. Cheng: Int. Sch. Scient. Res. Inno. 8 (2014) 1215.

10 C. J. Lin, H. T. Yau, C. Y. Lee, and K. H. Tung: IEEE/ASME Trans. Mech. 18 (2013) 1691.

11 C. J. Lin, C. Y. Lee, and Y. Liu: Appl. Sci. 7 (2017) 707.

12 C. Hirunyapruk, M. J. Brennan, B. R. Mace, and W. H. Li: Smart Mater. Struct. 19 (2010) 055020.

13 Y. Sun and M. Thomas: J. Vib. Control 17 (2011) 1253.

14 F. Weber and M. Maslanka: Smart Mater. Struct. 21 (2012) 055011.

15 S. B. Choi and Y. K. Park: J. Sound Vib. 172 (1994) 428.

16 L. A. Zadeh: IEEE Trans. Syst. Man Cybern. 3 (1973) 28.

17 L. A. Zadeh: Info. Sci. 8 (1975) 199.

18 E. H. Mamdani and S. Assilian: Int. J. Man Machine Stud. 7 (1975) 1.

19 E. H. Mamdani: IEEE Trans. Comp. 26 (1977) 1182.

20 J. M. Mendel: Proc. IEEE Fuzz. Conf. St. Louis, MO, 2003, May 26.

21 J. M. Mendel: IEEE Trans. Fuzzy Syst. 12 (2004) 84.

22 W. T. Thomson and M. D. Dahleh: Theory of Vibration with Applications (Taylor \& Francis, New York, 1993) p. 70.

\section{About the Authors}

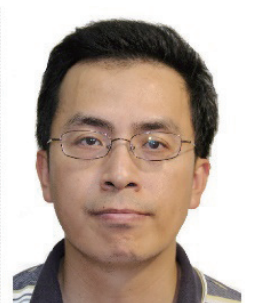

Chih-Jer Lin received his B.S., M.S., and Ph.D. degrees from the National Cheng Kung University, Tainan, Taiwan, in 1992, 1994, and 1998, respectively, all in mechanical engineering. He is currently a professor and the chairman of the Graduate Institute of Automation and Technology, National Taipei University of Technology, Taipei, Taiwan. His current research interests include mechatronics, vibration control, motion control, system identification, sliding-mode control, robotics, and evolutionary algorithms. Professor Lin has been one of the guest editors of Applied Sciences (ISSN 2076-3417) and Sensors (ISSN 1424-8220), and he is in the editorial board of the Journal of Chinese Society of Mechanical Engineers.

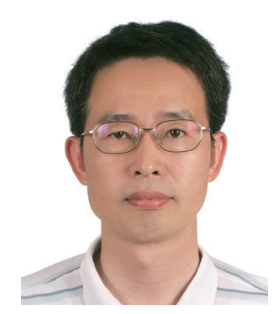

Chun-Ying Lee received his B.S. degree from the Department of Mechanical Engineering, National Taipei Institute of Technology, Taipei, Taiwan, in 1980, M.S. degree in mechanical engineering from National Sun Yat-sen University, Kaohsiung, Taiwan, in 1985, and Ph.D. degree in engineering mechanics from Michigan State University, East Lansing, MI, USA, in 1991. He is currently a professor of the Graduate Institute of Manufacturing Technology, National Taipei University of Technology, Taipei. His current research interests include smart materials, vibration control, and supercritical carbon-dioxide-assisted electroplating. 


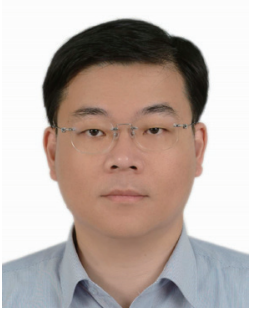

Chih-Keng Chen received his B.S. and M.S. degrees in mechanical engineering from National Cheng-Kung University, Tainan, Taiwan, in 1986 and 1988, respectively, and Ph.D. degree in systems and control engineering from Case Western Reserve University, Cleveland, OH, USA, in 1993. He is currently an associate professor of the Department of Vehicle Engineering, National Taipei University of Technology, Taipei, Taiwan. His current research interests are hydraulic systems, vehicle dynamics and control, and vehicle braking control systems.

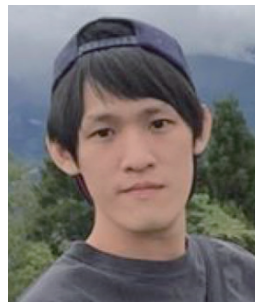

Yong-Sheng Li received his B.S. and M.S. degrees in automation technology from National Formosa University and National Taipei University of Technology, Taiwan, in 2013 and 2015, respectively. From 2015 to 2016, he fulfilled his mandatory military service. He is currently an engineer in UDE Corp. His research interests are in mechatronics and vibration control. 